\title{
EFEITO DE DIFERENTES CONDIÇÕES DE FERMENTAÇÃO SOBRE O TEOR E COMPOSIÇÃO DA FRAÇÃO DE SAPOGENINAS DO SUCO DE SISAL ${ }^{(1)}$
}

\author{
MARCo ANTONIO TEIXEIRA ZULLO (2), ROBERTO MACHADO DE MORAES, Segea a de \\ Fitoquimica, ANTONIO LUIZ DE BARROS SALGADO e ANiSIO AZZINI (2), Seção de \\ Plantas Fibrosas, Instituto Agronòmico.
}

\section{RESUMO}

Suco de sisal (Agave sisalana (Engelm.) Perrine), recém-coIhido, foi deixado fermentar por dez dias, espontaneamente ou por Sacharomyces cerevisae, processando-se, em cada caso, a fermentação protegida ou não do ambiente. Observou-se que os teores de sapogeninas totais, hecogenina, tigogenina, de duas sapogeninas não-identificadas, e de sólidos totais são significativamente dependentes do tipo e da duração da fermentação, sendo a última característica dependente também da condição de fermentação. Obtiveram-se maiores teores de sólidos totais e de sapogeninas totais por fermentação espontânea que por S. cerevisae e, em ambos os casus, quando protegida do ambiente. Não há, entretanto, qualquer correlação significativa entre os teores de sólidos totais e de sapogeninas. As reaçōes de formação de sapogeninas são oscilantes, fazendo que os maiores teores de sapogeninas sejam obtidos em torno do segundo dia de fermentação.

(1) Trabalho apresentado na 35a Reuniāo Anual da Sociedade Brasileira para o Progresso da Ciência, realizada em Belém (PA) a 6-13 de julho de 1983. Recebido para publicação a 27 de abril de 1983.

(2) Com bolsa de suplementação do CNPq. 


\section{INTRODUÇĀO}

Desde o reconhecimento do suco de sisal como uma fonte barata e abundante de hecogenina (3), um dos precursores mais adequados à síntese de corticosteróides (6), há relatos de que tanto esta sapogenina quanto a tigogenina podem ser encontradas na fração de sólidos do suco $(3,5,11)$. Segundo a literatura, os melhores métodos para a obtenção de sapogeninas a partir do suco de sisal envolvem sua fermentação espontânea por cerca de dez dias, período em que ocorre a decantação dos sólidos presentes, e extração das sapogeninas da porção de sólidos obtida $(10,11)$. Um ponto levantado contra esse uso de processos fermentativos é que, no seu decorrer, há a maior produção de x'ockogenina (1) - o diidroderivado de hecogenina naturalmente presente no suco de sisal (2) - o que reduz o valor comercial de concentrados de sisal (coffee grounds) utilizados para a extração de hecogenina. Com o fim de esclarecer a situação exposta, foram estudados os efeitos de diferentes condições de fermentação, espontânea e por Saccharomyces cerevisae, do suco de sisal sobre o teor de sólidos totais e de suas sapogeninas, em especial hecogenina e tigogenina.

\section{MATERIAL E MÊTODOS}

Folhas de sisal (Agave sisalana (Engelm.) Perrine), cultivado no Centro Experimental de Campinas, Instituto Agronômico, foram colhidas e imediatamente prensadas em moenda de cana. As fibras e mucilagens foram desprezadas e o suco, representando cerca de $54 \%$ do peso fresco das folhas, recolhido.

Cada $250 \mathrm{ml}$ de suco foi colocado em um frasco de erlenmeyer de $500 \mathrm{ml}$ e deixado fermentar segundo cada um dos quatro tratamentos, em duplicata: 1) fermentação espontânea; 2) fermentação espontânea protegida do ambiente; 3) fermentação por S. cerevisae; 4) fermentação por S. cerevisae protegida do meio ambiente. A levedura, nos tratamentos 3 e 4 , foi adicionada na taxa de $1 \%$ em peso ao suco recém-extraído. A proteção do meio ambiente foi realizada com auxilio de tampōes de algodão e os frascos de fermentação foram ocasionalmente agitados durante os dez dias em que se acompanhou a fermentação.

A cada 24 horas, os sucos foram eficientemente homogeneizados e, imediatamente, tomadas alíquotas de $10 \mathrm{ml}$, que, após secas em estufa a $60^{\circ} \mathrm{C}$, por 24 horas, com ventilação forçada de ar, forneceram os teores de sólidos totais.

Os sólidos totais foram submetidos ao processo de CARLE \& REINHARDT (4) para a detecção e determinação de sapogeninas por cromatografia em fase gasosa. A quantificação dos teores de sapogeninas foi feita pelo uso de soluções padrōes de hecogenina e tigogenina, previamente obtidas. Foi utilizado cromatógrafo CG mod. 37-D, com detetor de ionização de chama, coluna de vidro de $2 \mathrm{~m} \times 3 \mathrm{~mm}$ empa- 
cotada com SE-30 a $2 \%$ sobre Chromosorb WAW-DMCS, cromatografia isotérmica a $260^{\circ} \mathrm{C}$.

As análises de variância foram efetuadas segundo o delineamento de blocos inteiramente casualizados, considerando-se as variáveis seguintes: tipo (espontânea ou por S. cerevisae), condição (protegida ou não do ambiente) e duração da fermentação. As curvas mostrada nas figuras 1 e 2 foram obtidas por regressão polinomial, de modo a maximizar os coeficientes de determinação.

\section{RESULTADOS E DISCUSSÃo}

Durante os processos fermentativos, acompanhou-se a variação dos teores de sólidos totais do suco de sisal (Figura 1). Observou-se que nos processos de fermentação espontânea, eles se mantiveram aproximadamente constantes durante os dez primeiros dias de fermentação, enquanto nos processos em que se empregou S. cerevisae, tais teores decresceram no mesmo período de tempo. Segundo análise de variância, o teor de sólidos totais é dependente do tipo, da condição e da duração do processo fermentativo, sendo que a fermentação espontânea protegida do meio ambiente é significativamente superior a outros processos, quanto a esse aspecto. Não foi possivel encontrar qualquer correlação significativa entre os teores de sólidos totais e os de sapogeninas totais, hecogenina ou tigogenina.

No caso presente, pôde-se acompanhar, a grosso modo, a dinâmica de sintese e degradação de apenas quatro das sapogeninas presentes no sisal (2): hecogenina, tigogenina e duas sapogeninas não identificadas. Os tempos de retenção absolutos $\left(t_{R}\right)$ e relativos $\left(t_{R R}\right)$ das quatro sapogeninas detectadas encontram-se no quadro 1. A soma dos teores das quatro sapogeninas, em cada caso, forneceu os teores de sapogeninas totais.

QUADRO 1. Sapogeninas esteroidicas detectadas no suco de sisal, por cromatografia em fase gasosa ${ }^{2}$

\begin{tabular}{|c|c|c|}
\hline Sapogeninas & $t_{R}$ & $t_{R R}$ \\
\hline & $\min$ & \\
\hline Tígogenina & $4,21 \pm 0,66$ & 1,00 \\
\hline Hecogenina & $7,22 \pm 1,52$ & $1,64 \pm 0,02$ \\
\hline $\begin{array}{l}\text { 1* Sapogenina } \\
\text { não-identificada }\end{array}$ & $3,10 \pm 0,74$ & $0,72 \pm 0,03$ \\
\hline $\begin{array}{l}\text { 2* Sapogenina }^{\text {não-identificada }}\end{array}$ & $10,51 \pm 1,36$ & $2,56 \pm 0,06$ \\
\hline
\end{tabular}

(a) Cromatografia isotérmica a $260^{\circ} \mathrm{C}$, em coluna de vidro de $2 \mathrm{~m} \times 3 \mathrm{~mm}$, empacotada com SE-30 a $2 \%$ sobre Chromosorb WAW-DMCS. 


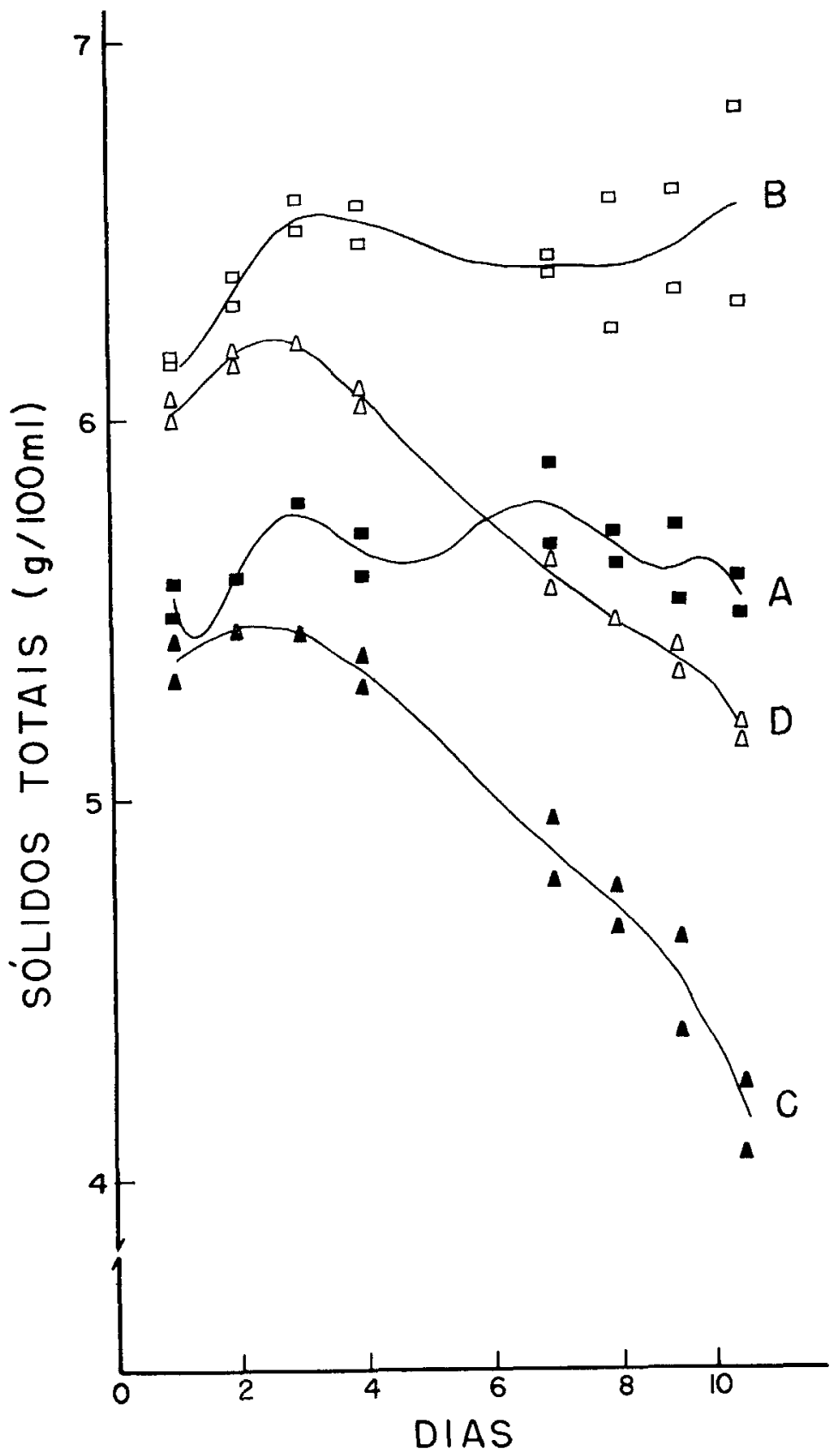

FIGURA 1. Variação do teor de sólidos totais durante a fermentação do suco de sisal - fermentação espontânea: A: em aberto; B: protegida do ambiente; fermentação por S. cerevisae - C: em aberto; D: protegida do ambiente. 

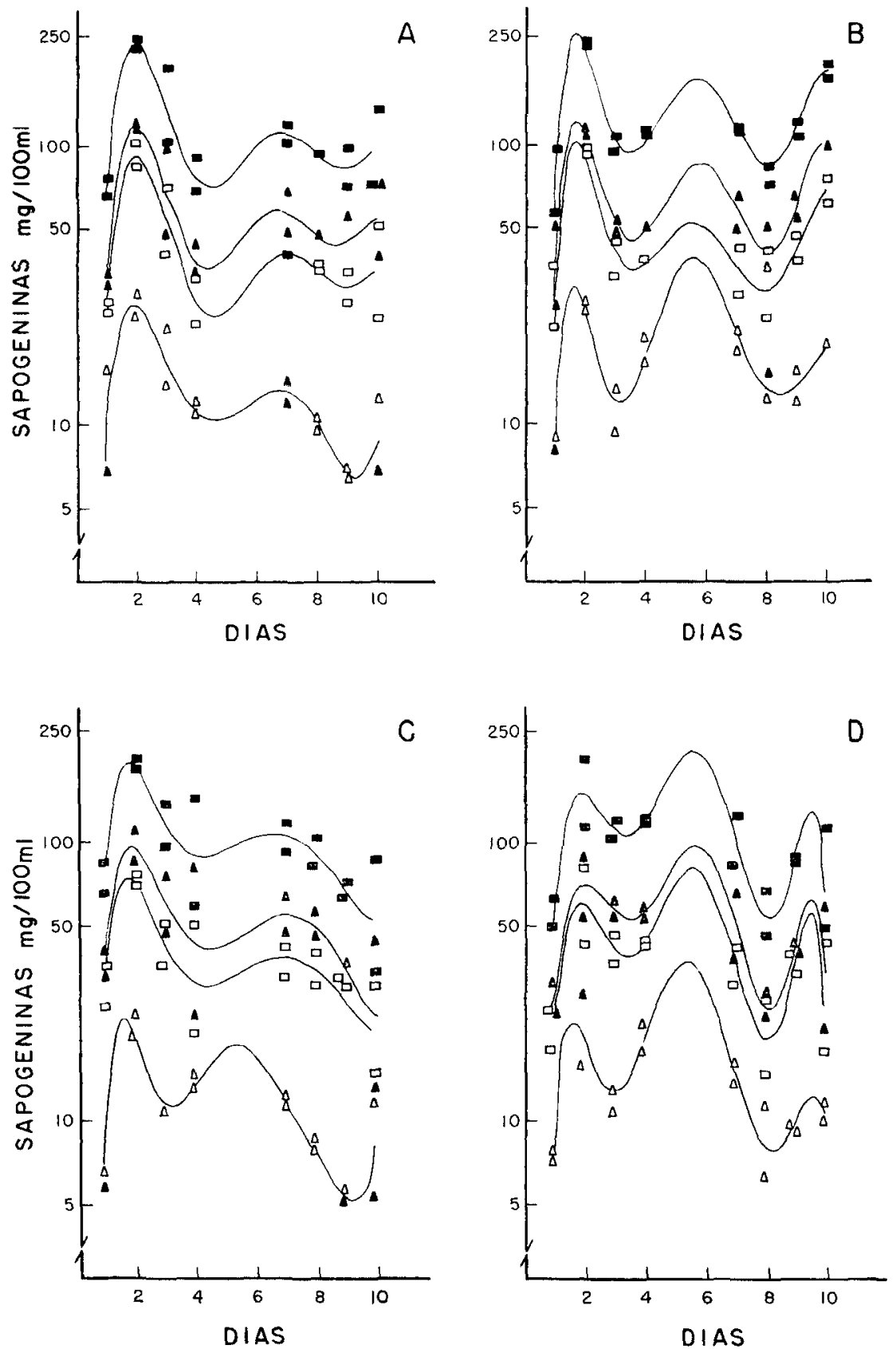

FIGURA 2. Variação dos teores de sapogeninas totais (a), de hecogenina ( $\square$ ), de tigogenina $(A)$ e de outras sapogeninas $(\Delta)$ durante a fermentaçào do suco de sisal (tratamentos conforme a Figura 1 ). 
O acompanhamento dos teores de sapogeninas totais, hecogenina e tigogenina, durante os processos fermentativos, mostrou uma seqüência de máximos e mínimos destes teores (Figura 2). Estes teores são dependentes do tipo e da duração do processo fermentativo, mas não da condição de fermentação utilizada, conforme avaliado por análise de variância. Os teores máximos absolutos do total de sapogeninas se situam entre 240 e $250 \mathrm{mg} / 100 \mathrm{ml}$ de suco de sisal para os processos de fermentação espontânea, e entre 170 e $200 \mathrm{mg} / 100 \mathrm{ml}$ de suco de sisal para os processos de fermentação por $\mathbf{S}$. cerevisae. De modo geral, esses máximos ocorrem próximo ao segundo dia de fermentação. A superioridade do processo de fermentação espontânea na produção de sapogeninas apenas reflete a inadequação do sistema enzimático da levedura para a metabolização deste tipo de substâncias, de vez que seu metabolismo esteroídico envolve a síntese, interconversão e degradação de ergosterol e derivados ( $(\boldsymbol{y})$.

Um exame da Figura 2 sugere a operação de um mecanismo básico, ainda que complexo, sobre a disponibilidade de sapogeninas ao meio. Como regra geral, essas sapogeninas existem nas plantas sob as formas livre ou, predominantemente, de glicosídios (saponinas) (9), embora no caso em questão ainda não se saiba a estrutura destes últimos. Nas fermentaçōes aqui utilizadas pode ocorrer uma série de reaçōes enzimáticas que promova, simultaneamente, a sintese e a interconversão das geninas, a sintese, a interconversão e a hidrólise das saponinas, bem como a degradação das substâncias envolvidas. Desta forma, a lei de velocidade de formação das sapogeninas, individualmente (e, por extensão, das sapogeninas totais), será não-linear. Havendo retroalimentação (feedback) sobre uma ou mais das reações envolvidas, como é o caso das interconversōes de geninas ou saponinas, a concentração das sapogeninas, individualmente e no total, variará não-monotonicamente ao longo da fermentação, fazendo que a reação de formação de sapogeninas seja oscilante (8), o que concorda com os dados aqui obtidos e explica a ocorrência de "fermentações secundárias" do suco de sisal citadas por MORS \& SHARAPIN (10). O erro experimental relativamente elevado, aqui obtido, não permite esclarecer, entretanto, se essas reações de formação de sapogeninas são oscilantes periódicas ou não.

\section{CONCLUSõeS}

1) O teor de sólidos totais do suco de sisal foi significativamente maior quando submetido à fermentação espontânea que quando por $\mathbf{S}$. cerevisae e, em ambos os casos, foi maior quando a fermentação foi conduzida protegida do meio ambiente que quando exposta ao ar;

2) Não houve qualquer correlação significativa entre os teores de sólidos totais e os teores de sapogeninas totais, hecogenina ou tigogenina;

3) Os teores de sapogeninas totais, hecogenina e tigogenina se mostraram dependentes do tipo e da duração da fermentação, mas não 
da condição de fermentaçāo, enquanto os teores de sóliclos totais foram dependentes das três variáveis estudadas;

4) As reações de formação de sapogeninas são reações oscilantes, o que faz que as concentrações de sapogeninas totais, hecogenina e tigogenina decresçam não-monotonicamente ao longo das fermentações, independentemente do tipo e das condições de fermentação utilizados;

5) Os teores máximos absolutos de sapogeninas totais, hecogenina e tigogenina ocorreram geralmente em torno do segundo dia de fermentação.

\section{AGRADECIMENTOS}

A Silvana Marques da Silva, estagiária, Sônia Aparecida Ferraz de Campos e Helena Kiyomi Ito, técnicas da Seção de Fitoquímica, pelo auxilio nas análises efetuadas. Ao Dr. Toshio Igue, da Seção de Técnica Experimental e Cálculo, pelo auxilio nas análises estatísticas.

\section{SUMMARY}

\section{THE EFFECT OF DIFFERENT FERMENTATION CONDITIONS ON THE YIELD AND COMPOSITION OF THE SAPOGENIN FRACTION OF SISAL JUICE}

Freshly collected sisal (Agave sisalana (Engelm.) Perrine) juice was fermented during ten days, spontaneously or by Saccharomyces cerevisae, either protected or unprotected from the environment. It was observed that the total sapogenins, hecogenin, tigogenin, two unidentified sapogenins, and total solids content were significantly dependent on the kind and duration of fermentation, the last characteristic being also dependent on the condition of fermentation. The higher total solids and sapogenins contents were obtained by spontaneous rather than by $\mathbf{S}$. cerevisae fermentation, and, in both cases, under protection from the environment. There was, however, no significant correlation between total solids and sapogenins contents. The sapogenin forming reactions are oscillating reactions, in such a manner that the better yield of sapogenins are obtained at about the second day of fermentation.

\section{REFERENCIAS BIBIMOGRAFICAS}

1. BLUNDEN, G.; JAFFER, J, A.; JEWERS, K.; BARBOUR, J. Rockogenin formation during the processing of Agave sisalana leaves for hecogenin. Lloydia, 40:218-219, 1977.

2. - $;$ YI, Y.; JEWERS, $K$. A reinvestigation of the steroidal sapogenins of Agave Sisalana. Lloydia, 37:10-16, 1974.

3. CALLOW, R. K.; CORNFORTH, J. W.; SPENSLFY, P. C. A source of hecogenin. Chemistry and Industry, 33:699-700, 1951.

4. CARLE, R. \& REINHARDT, E. Simultaneous determination of solasodine, diosgenin and tigogenin. Planta Medica, 38:381-383, 1980.

5. EDWARDS, M. G. Pilot scale manufacture of hecogenin from sisal waste. The East African Agricultural Journal, 18(1):21-23, 1952. 
6. FIESER, L. F. \& FIESER, M. Steroids. New York, Reinhold Publishing Co., 1959. 19-23, p.667-671.

7. FRYBERG, M.; OEHLSCHLAGER, A. C.; UNRAU, M. Biosynthesis of ergosterol in yeast. Evidence for multiple pathways. Journal of the American Chemical Society, 95:5747-5757, 1973.

8. HIGGINS, J. The theory of oscillating reactions. Industrial Engineering Chemistry, $59(5): 18-62,1967$.

9. MAHATO, S. B.; GANGULY, A. N.; SAHU, N. P. Steroid saponins. Phytochemistry, $21: 959-978,1982$.

10. MORS, W. B. \& SHARAPIN, N. A obtenção dos esteróides no sisal. Revista Brasileira de Tecnologia, São Paulo, 4:153-165, 1973.

11. SPENSLEY, P. C. A source of hecogenin. Chemistry and Industry, 19: $426-428,1952$. 